Check for updates

Cite this: RSC Adv., 2017, 7, 38434

Received 9th June 2017

Accepted 23rd July 2017

DOI: $10.1039 / \mathrm{c} 7 \mathrm{ra06449g}$

rsc.li/rsc-advances

\section{Antibacterial and cytocompatible AgNPs constructed with the assistance of Mefp-1 for orthopaedic implants $\uparrow$}

\author{
Yun Yang, ${ }^{\text {ab }}$ Yanmei Zhang, ${ }^{\text {ab }}$ Ren Hu, ${ }^{a}$ Qiaoling Huang, ${ }^{\text {ac }}$ Ke Wu, ${ }^{* d}$ Lihai Zhang, ${ }^{e}$ \\ Peifu Tang*e and Changjian Lin iD *ab
}

\begin{abstract}
The increasing threat of orthopedic implant failure caused by infection and loosening intensifies the need for novel surface functional treatment. In this study, a thin mussel adhesive protein (Mefp-1)/silver nanoparticle (AgNP) composite film constructed on titania nanotubes (TNTs) via a simple dip-coating method has been demonstrated. The TNT/Mefp-1/AgNP coating exhibits both high antibacterial activity and adequate cytocompatibility. The adherent Mefp-1 film could promote preosteoblast proliferation and reduce AgNP-induced cytotoxicity. The AgNPs $(\sim 10 \mathrm{~nm})$ constructed with the assistance of Mefp-1 are effective for the elimination of both Gram-positive Staphylococcus aureus (S. aureus) and Gramnegative Escherichia coli (E. coli) via a combination of contact-killing and release-killing modes. This facile and mild functionalization strategy exhibits promising applications in surface antibacterial modifications, especially in three-dimensional sophisticated medical devices.
\end{abstract}

\section{Introduction}

Orthopedic implants (OIs), such as screw nails, plates, intramedullary rods, and prosthetic hip, are being successfully and widely applied in the field of orthopedic surgery. However, OI failure frequently caused by infection has become one of the worst outcomes of orthopedic intervention in terms of both patient prognosis and healthcare cost. ${ }^{1,2}$ To improve the success of medical implants, it is urgent to endow the various implants with surface properties of good bone tissue integration and concomitant antibacterial ability. The success or failure of OIs largely depends on the interaction between cells and implant surface after implantation. Many studies have been focused on improving the osseointegration and antimicrobial activity of the orthopedic devices transplanted in the human body. Current surface functional modification includes (i) construction of

${ }^{a}$ State Key Laboratory of Physical Chemistry of Solid Surfaces, College of Chemistry and Chemical Engineering, Xiamen University, Xiamen, China. E-mail: cjlin@xmu. edu.cn

${ }^{b}$ Beijing Engineering Laboratory of Functional Medical Materials and Devices, Beijing Medical Implant Engineering Research Center, Beijing Naton Technology Group Co. Ltd, Beijing, China

${ }^{c}$ Research Institute for Biomimetics and Soft Matter, Department of Physics, School of Physics and Mechanical \& Electrical Engineering, Xiamen University, Xiamen, China ${ }^{d}$ Department of Cardiology, The Affiliated Dongnan Hospital of Xiamen University, Zhangzhou, China.E-mail: wukeyyun@163.com

${ }^{e}$ Department of Orthopaedics, General Hospital of Chinese PLA, Beijing, China. E-mail: pftang301@163.com

$\dagger$ Electronic supplementary information (ESI) available. See DOI: 10.1039/c7ra06449g micro-nano structures via sandblasting/acid etching, anodic oxidation, and micro-arc oxidation; $;^{3-5}$ (ii) bioactive coatings such as calcium phosphate bioceramic and macromolecules with multitudinous biological activity; ${ }^{6-8}$ and (iii) antibacterial coatings loaded with antimicrobials, inorganic antibacterial agents, and antimicrobial peptides. ${ }^{9-12}$ However, it remains a big challenge to endow an orthopedic implant with two properties, i.e. excellent bioactivity and effective antibacterial ability which are a pair of contradiction and conflict.

Silver is well-known for its remarkable antimicrobial activity against a broad spectrum of pathogens including both Grampositive and Gram-negative bacteria. Silver nanoparticles (AgNPs) exhibit a much stronger antibacterial effect than regular metallic silver due to their large specific area for bacterial interaction and a size-dependent release of silver ions. ${ }^{13}$ AgNP coatings have been already used in a variety of medical products such as urinary catheters and suture threads. ${ }^{\mathbf{1 4 , 1 5}}$ Various approaches such as ultraviolet irradiation, electrochemical deposition, and plasma immersion ion implantation have been previously reported for the preparation of a AgNP coating. ${ }^{16-18}$ Recently, AgNPs generated using polydopamine (a material from mussel-inspired chemistry) have experienced a considerable achievement in diverse fields such as in antibacterial surface modification on hydrogels, ${ }^{19}$ cottons, ${ }^{20}$ metals, ${ }^{21,22}$ and polymers. ${ }^{23-25}$ However, the possible cytotoxicity of AgNPs remains a concern when they are used in various OI devices.

Marine mussels are able to strongly adhere onto a wide range of materials even under wet marine conditions. The adhesion of mussel is rapid and versatile owing to the adhesive proteins, 
such as Mytilus edulis foot proteins (Mefp), secreted by it. ${ }^{26}$ These proteins contain a high level of unusual amino acid and dihydroxyphenylalanine (DOPA) that may confer the observed adhesive properties. ${ }^{27}$ Nowadays, commercially available Mefps and related products have been widely used in various fields such as in corrosion protection for stainless steel and tissue adhesive. ${ }^{28,29}$ As a strong adhesive polyphenolic protein extract from Mytilus edulis, Cell-Tak ${ }^{\mathrm{TM}}$ (BD diagnostics) has been widely used to attach cells or tissue sections to various types of surfaces including plastic, glass, metal, FEP polymer, and biological materials. $^{30}$ Compared to polydopamine-based antibacterial coating, it is more interesting to study the performance of Mefps-based coating for antibacterial surface modification due to its unique powerful functionality and biological natural properties.

With the rapid development of nanotechnology, various forms of nanostructures, such as nanoparticles, nanotubes, nanobelts, nanorods, and nanowires, with potential uses in biorelated applications have been successfully fabricated. ${ }^{31-34}$ Among these, highly ordered titania nanotubes (TNTs) have gained significant attention in orthopaedic applications due to their unique properties such as large surface area, high corrosion resistance, non-magnetic, good biomechanical compatibility, as well as inherent biocompatibility allowing great cell adhesion, proliferation, and mineralization. ${ }^{35}$ Thus, the smart TNT surface could provide a better template for bone cell growth, osteoblasts activity, and sustainable drug delivery than the titanium surface. ${ }^{36,37}$

Herein, the motivation of this study was to explore the antimicrobial efficacy and biological safety of AgNPs onto TNTs assisted by Mefp-1 coatings. TNTs were used as a platform for AgNP loading and silver ion release and fabricated through a facile anodic oxidation process. Among the proteins in the Mefps family, Mefp-1 has been first purified and characterized. It is a large and basic hydrophilic protein consisting of a large number of repetitive decapeptide units. ${ }^{38}$ Nanolayered Mefp-1 coating was spontaneously formed on the TNT inner and outer surface via a simple immersion method. The Mefp- 1 coating is capable of chelating and reducing silver ions into nanoparticles with a relatively small diameter of about $10 \mathrm{~nm}$ and stabilizing them firmly onto the surface. Furthermore, the biocompatibility, cytotoxicity, and antimicrobial activity of these Mefp-1modulated AgNP@TNTs were assessed in detail to facilitate clinical applications of the effective OIs with a AgNP@TNT film.

\section{Materials and methods}

\subsection{Preparation of the Mefp-1/AgNP coating}

Commercial pure titanium (Ti) foils $(1 \mathrm{~mm} \times 10 \mathrm{~mm} \times 10 \mathrm{~mm})$ were polished and ultrasonically cleaned in acetone, ethanol, and deionized (DI) water before use. Titanium dioxide $\left(\mathrm{TiO}_{2}\right)$ nanotube array films were fabricated in a $0.5 \mathrm{wt} \%$ hydrofluoric acid electrolyte at $20 \mathrm{~V}$ for $20 \mathrm{~min}$ (denoted as TNT). The mussel adhesive protein Mefp-1 (Biopolymer Products AB, Gothenburg, Sweden) was dissolved in $1 \%$ citric acid. The concentration of Mefp-1 was $1 \mathrm{mg} \mathrm{ml}^{-1}$, and the $\mathrm{pH}$ of Mefp-1 solution was adjusted to 4.6 using $1 \mathrm{M} \mathrm{NaOH}$. The TNT sheets were treated with an Mefp-1 solution at room temperature for $1 \mathrm{~h}$ and subsequently washed with DI water and dried at $60{ }^{\circ} \mathrm{C}$ for $1 \mathrm{~h}$ (denoted as MTNT). The MTNT sheets were then immersed in $\mathrm{AgNO}_{3}$ solutions at five different concentrations (1, 0.1, 0.01, 0.001, and 0.0001 M, denoted as MTNT-Ag1, MTNT-Ag2, MTNTAg3, MTNT-Ag4, and MTNT-Ag5, respectively) under agitation (160 revolutions per minute) at room temperature for $4 \mathrm{~h}$. The MTNT-Ag samples were ultrasonically cleaned in DI water and then dried in a vacuum oven.

\subsection{Physicochemical characterization}

The surface topography was observed using scanning electron microscopy (FE-SEM, Hitachi S4800, Japan) with an accelerating voltage of $15 \mathrm{kV}$. The surface characteristics and roughness of the films were evaluated using atomic force microscopy (AFM, CSPM5000, China) with silicon tip probe of a spring constant of $40 \mathrm{~N} \mathrm{~m}^{-1}$ and a resonant frequency of $300 \mathrm{kHz}$ at the tapping mode with a sweep speed of $1 \mathrm{~Hz}$. The obtained images were analyzed via CSPM Imager Analysis. The microstructure of the AgNP-loaded nanotubes was determined by transmission electron microscopy (TEM, JEM-2100, Japan). The coating was scraped and ultrasonically dispersed in ethanol. The suspension was dropped on TEM grids and allowed to dry before analysis. Surface chemical compositions were measured via Xray photoelectron spectroscopy (XPS, VG ESCALAB MKII) with an $\mathrm{Al} \mathrm{K} \alpha \mathrm{X}$-ray source (1486.6 eV). The binding energy were referenced to the $\mathrm{C}(1 \mathrm{~s})$ hydrocarbon peak at $285.0 \mathrm{eV}$. Raman spectra were obtained using Raman spectroscopy (XploRA, Horiba JobinYvon, France) in the wavelength range from 50 to $2000 \mathrm{~cm}^{-1}$ with a $532 \mathrm{~nm}$ air-cooled argon ion laser. The static water contact angles (CA) were measured using an optical contact angle meter system (Dataphysics, OCA-20) at ambient temperature and humidity. The DI water droplets used for the CA measurement were $3 \mu \mathrm{l}$, and three measurements were performed on each sample for statistical accountability. A total of three samples were measured for each group.

\subsection{Silver ion release}

The treated MTNT-Ag coating was immersed in $3 \mathrm{ml}$ phosphate buffered saline (PBS, $\mathrm{pH} 7.4$ ) solution at $37{ }^{\circ} \mathrm{C}$. At each time point $(1,2,3,5,8,10$, and 14 day), the entire volume was obtained, and fresh PBS was refilled accordingly. The concentration of the released silver was quantified using inductively coupled plasma mass spectrometry (ICP-MS, Thermo X series II).

\subsection{Antibacterial study}

Staphylococcus aureus (S. aureus, ACTT) and Escherichia coli (E. coli, ACTT) strains were grown overnight in a Luria-Bertani (LB) medium at $37{ }^{\circ} \mathrm{C}$. Bacterial suspension $\left(105 \mathrm{CFU} \mathrm{ml}^{-1}, 100 \mu \mathrm{l}\right)$ were uniformly dispersed on the surface of the LB plates. The sterile samples (with a diameter of $8 \mathrm{~mm}$ ) were laid on the top of the plate and incubated at $37{ }^{\circ} \mathrm{C}$ for $12 \mathrm{~h}$. The diameters of zones of inhibition (ZoI) against $S$. aureus and E. coli were measured. For the antibacterial efficiency test, samples were immersed in $1 \mathrm{ml}$ bacterial suspension (106 CFU ml $\mathrm{m}^{-1}$ ) and then shaken at $200 \mathrm{rpm}$ at $37^{\circ} \mathrm{C}$ for $6 \mathrm{~h}$. Subsequently, the 
bacterial solution was obtained, and the number of total viable counts of each sample was calculated through the flat colony counting method. The bactericidal efficiency was calculated as follows: $[(A-B) / A] \times 100 \%$, where $A$ and $B$ are the CFU counts for Ti and MTNT-Ag, respectively. The morphology of bacteria adhered to the samples was observed via SEM.

\subsection{Biocompatibility evaluation}

Mouse pre-osteoblasts MC3T3-E1 cells (Cell Resource Center, PUMC, China) were cultured in an alpha minimum essential medium ( $\alpha$-MEM) supplemented with $10 \%$ FBS at $37{ }^{\circ} \mathrm{C}$ in a humidified incubator with $5 \% \mathrm{CO}_{2}$. The cells were seeded on the samples placed in a 24-well culture plate at a density of $1 \times$ 104 cells per well. After culturing for $1,3,5$, and 7 days, the cell viability was determined using WST-1 cell proliferation and cytotoxicity assay kit (Beyotime, China), and the morphology of the cells on the samples were observed by SEM. The alkaline phosphatase (ALP) activity was measured using an ALP kit (Jiancheng Bioengineering Institute, China). After 10 days of culture, the cells on the samples were lysed in $1 \%$ Triton X-100, and the absorbance of the supernatant at $520 \mathrm{~nm}$ was measured using a microplate reader (BioTek-ELx800, China); in addition, the ALP activity was normalized to the total protein content. The cytoskeleton organization of cells grown on the samples was analyzed using filamentous actin (F-actin) staining. After 1 day of culture, cells were fixed with $2.5 \%$ glutaraldehyde for $20 \mathrm{~min}$, stained with phalloidin-tetramethylrhodamine B isothiocyanate (phalloidin-TRITC, Sigma, USA) for $1 \mathrm{~h}$, and further stained with DPAI for $5 \mathrm{~min}$ at room temperature in the dark. The stained cells were observed via laser confocal microscopy (TCSSP5, Leica Microsystems, Germany).

\subsection{Statistical analysis}

Statistical analysis of the experimental data was performed using SPSS 16.0. Data have been expressed as the mean \pm standard deviation (SD). Analysis of the results was carried out using Student's $t$-test, with a significance level of $P<0.05$.

\section{Results}

\subsection{Fabrication and characterization of the Mefp-1/AgNP/ TNT coatings}

As illustrated in Fig. 1, the Mefp-1/AgNP/TNT coatings on Ti were synthesized via a mild and convenient approach including anodic oxidation, Mefp-1 self-polymerization, and autooxidization, and in situ reduction of AgNPs. Mefp-1, with a molecular weight of $108 \mathrm{kDa}$, consists of various (75-80) repetitive decapeptide units and contains a number of unusual amino acids, such as lysine, and high level (10-15 mol\%) of dihydroxyphenylalanine (DOPA).$^{38}$ After anodic oxidation in a HF solution for $20 \mathrm{~min}$, the typical TNT array structure had a diameter of $\sim 80 \mathrm{~nm}$ and length of $\sim 300 \mathrm{~nm}$ (Fig. 2 and $3 \mathrm{a}$ ). The wall thickness of the nanotubes was about $5 \mathrm{~nm}$, which increased to $\sim 15 \mathrm{~nm}$ after the nanotubes were coated with the Mefp-1/AgNP film.

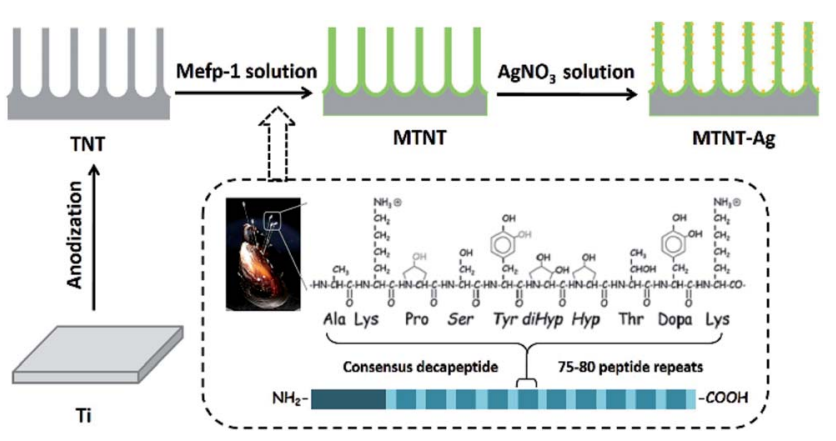

Fig. 1 Schematic of the procedure for the preparation of Mefp-1/ AgNP coating.

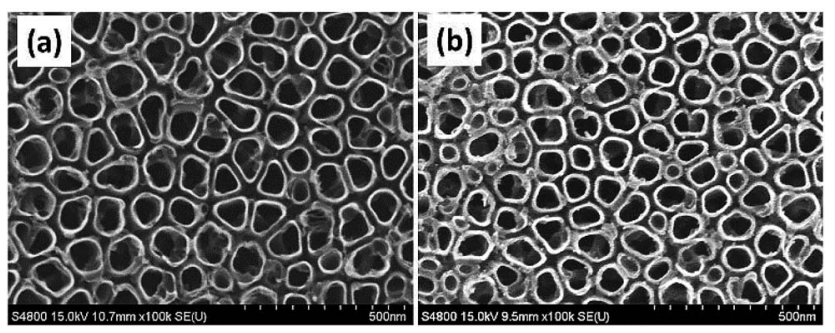

Fig. 2 SEM images of (a) TNT and (b) TNT/Mefp-1/Ag1.

The nanotubular structure was well preserved after surface modification, and highly dispersed AgNPs were incorporated onto the nanotube wall (Fig. 3a, indicated with red arrows). The AgNPs showed a durable firm adhesion, even after being treated with ultrasonic cleaning for $10 \mathrm{~min}$ (data not shown). The AgNPs attached to the TNT wall have a diameter of $\sim 10 \mathrm{~nm}$. After treatment with different concentrations of silver nitrate solution, the grain size and morphology exhibited no obvious change (Fig. 3b). The AFM images indicated that the MTNT and MTNT-Ag surfaces had similar roughness (Fig. 3c).

The chemical states of the samples were analyzed by XPS. The successful oxidation polymerization of Mefp- 1 on the TNTs could be ascertained by comparing the XPS spectra of the TNT before and after the deposition process. As shown in Fig. 4a and $\mathrm{b}$, the presence of the Mefp- 1 was confirmed by the appearance of $\mathrm{N}$ 1s peaks. High resolution XPS spectra were obtained to determine the chemical states of silver. The $\mathrm{Ag} 3 \mathrm{~d}$ peak at $368.5 \mathrm{eV}\left(\mathrm{Ag} 3 \mathrm{~d}_{5 / 2}\right)$ and $374.5 \mathrm{eV}\left(\mathrm{Ag} 3 \mathrm{~d}_{3 / 2}\right)$ revealed that silver existed in a metallic form. ${ }^{16}$ The silver content in MTNT-Ag1, MTNT-Ag2, MTNT-Ag3, MTNT-Ag4, and MTNT-Ag5 determined from the XPS survey spectra was $6.39,3.14,2.74,1.74$, and 0.38 at\%, respectively (Table S1 $\dagger$ ). The wettability of the samples was measured via the sessile drop technique. Compared to the raw $\mathrm{Ti}$ foils with the water contact angle of $75.5 \pm 2.6^{\circ}$, MTNT and MTNT-Ag samples exhibited high hydrophilicity with water contact angles ranging from $10.7 \pm 3.7^{\circ}$ to $12.8 \pm 3.2^{\circ}$.

The chemical composition on the surface was further investigated by Raman spectroscopy. Silver is one of the best materials for inducing surface enhanced Raman scattering (SERS), and surface modification with AgNPs is apt to obtain strong SERS signals. As shown in Fig. 4c, the peak at $1330 \mathrm{~cm}^{-1}$ 
(a)
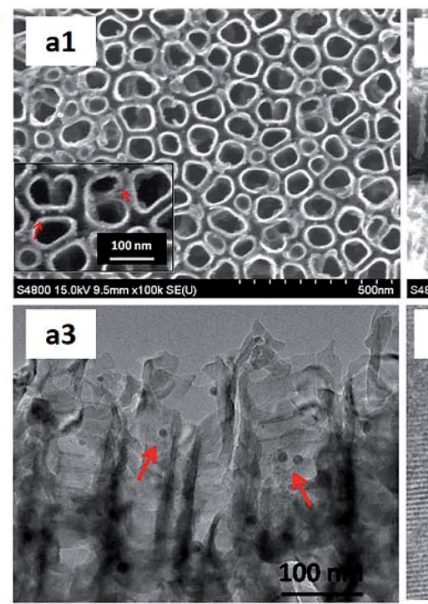

a2
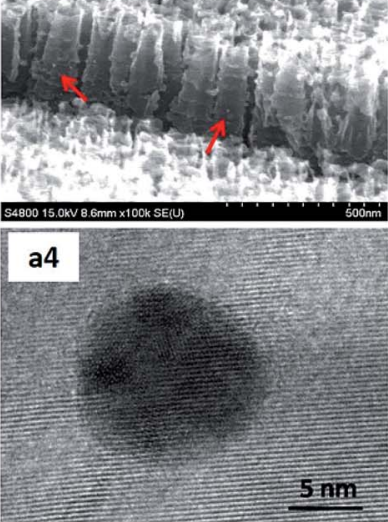

(b)

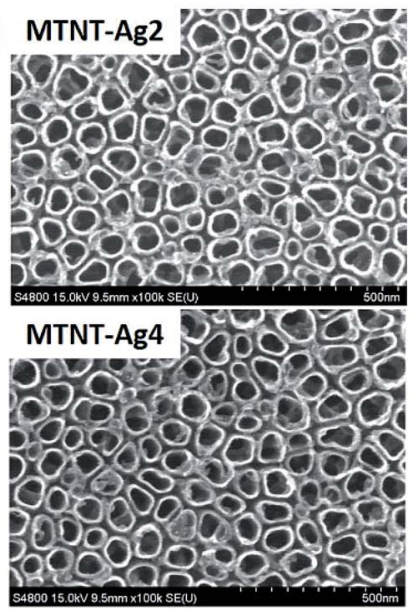

MTNT-Ag3

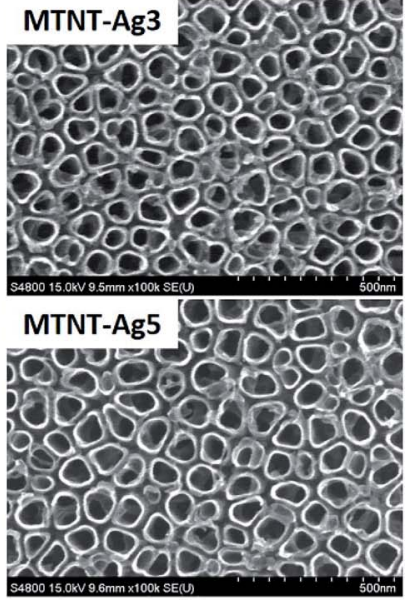

\section{(C) $\mathrm{Ti}$}
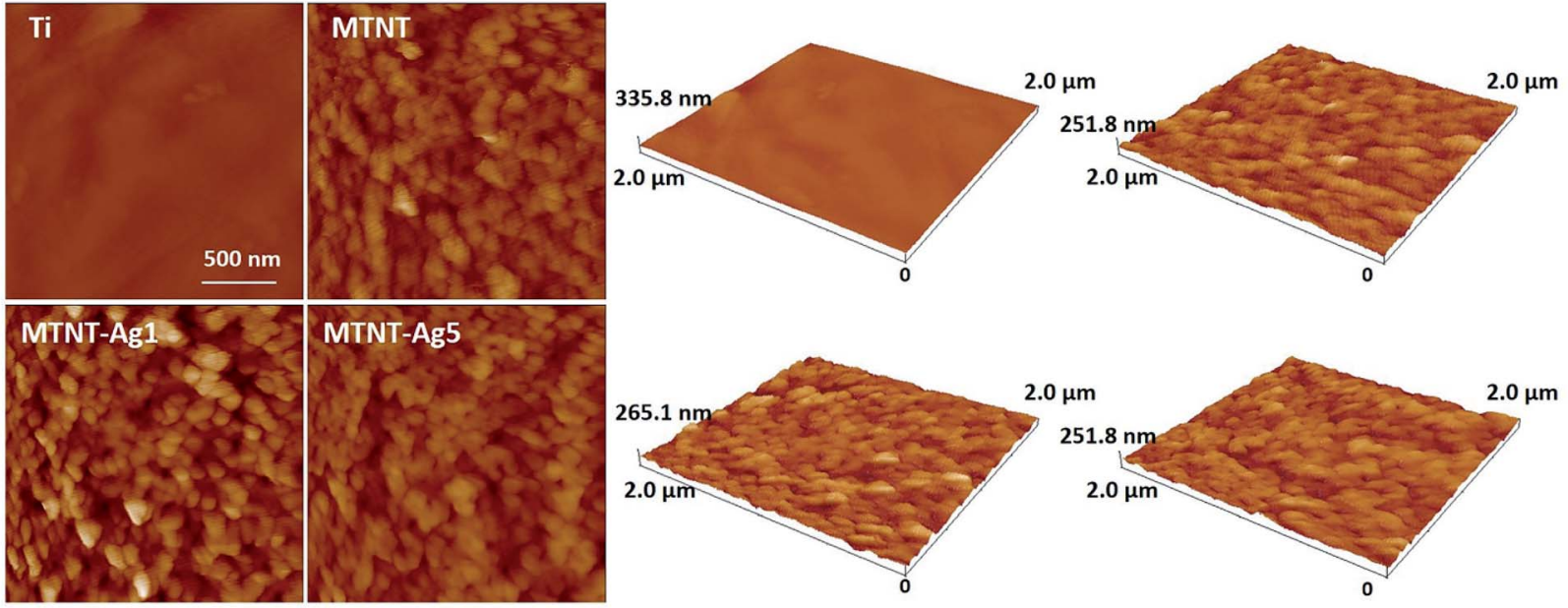

Fig. 3 Morphological characterization of the Mefp-1/AgNP coatings: (a) SEM and TEM images of MTNT-Ag1; (b) SEM images of MTNT-Ag2 to MTNT-Ag5; and (c) AFM images of Ti, MTMT, MTMT-Ag1, and MTNT-Ag5.

(amide III) was assigned to the vibrational mode of dopamine, which is due to the distortion of the $\mathrm{C}-\mathrm{C}$ bond connecting the two oxygen-bearing carbon atoms. The peak at $1595 \mathrm{~cm}^{-1}$ (amide II) was related to the in-plane $\mathrm{N}-\mathrm{H}$ bending and $\mathrm{C}-\mathrm{N}$ stretching of dopamine, both of which corresponded to the characteristic aromatic groups in polydopamine. ${ }^{24,39}$ No discernible peak of aromatic group was observed in the MTNT group. The MTNT-Ag groups showed an efficient SERS activity, and the intensities of the characteristic peaks increased with the AgNP dose (strongest in MTNT-Ag1).

Fig. 4d displays the silver release time profiles from MTNT$\mathrm{Ag}$ in PBS. In the early stage, the released silver ions decreased in the order of MTNT-Ag1, MTNT-Ag2, MTNT-Ag3, MTNT-Ag4, and MTNT-Ag5. Moreover, silver ions released from the MTNT-Ag samples decreased fast with time. After 5 days of immersion, only continuously releasing silver ions at a very low level could be detected.

\subsection{Antibacterial assessment}

The antibacterial properties of the MTNT-Ag samples were evaluated for both Gram-positive and Gram-negative bacteria. The Zol test is based on the leaching of silver ions from the material surface, and the inhibition of the bacterial growth depends on the sufficient concentration of silver ions in the surrounding aqueous environment. As demonstrated in Fig. 5a, the samples MTNT-Ag1, MTNT-Ag2, and MTNT-Ag3 showed a clear inhibition zone of both $S$. aureus and E. coli; this indicated an efficient wide spectrum antibacterial ability. Moreover, the MTNT-Ag4 and MTNT-Ag5 samples showed strong antibacterial effect on $E$. coli, whereas they exhibited only weak antibacterial effect on $S$. aureus. The diameters of the bacterial inhibition zone, as shown in Table S2, $\dagger$ demonstrated that the MTNT-Ag samples were more effective towards Gram-negative bacteria.

It was observed that the bacterial solution obviously became feculent after incubation for $6 \mathrm{~h}$ with Ti and MTNT; this indicated no antibacterial effect on $S$. aureus and E. coli in vitro. However, the MTNT-Ag groups showed significant antibacterial effects on both $S$. aureus and E. coli. The antimicrobial activities were positively correlated with the AgNP dose loaded on the MTNT surface. The bacterial suspension turbidity increased in the order of MTNT-Ag1, MTNT-Ag2, MTNT-Ag3, MTNT-Ag4, and MTNT-Ag 5 for both $S$. aureus and E. coli. For MTNT-Ag1, MTNTAg2, and MTNT-Ag3, all groups provided significant antibacterial effects on both Gram-positive and Gram-negative bacteria 

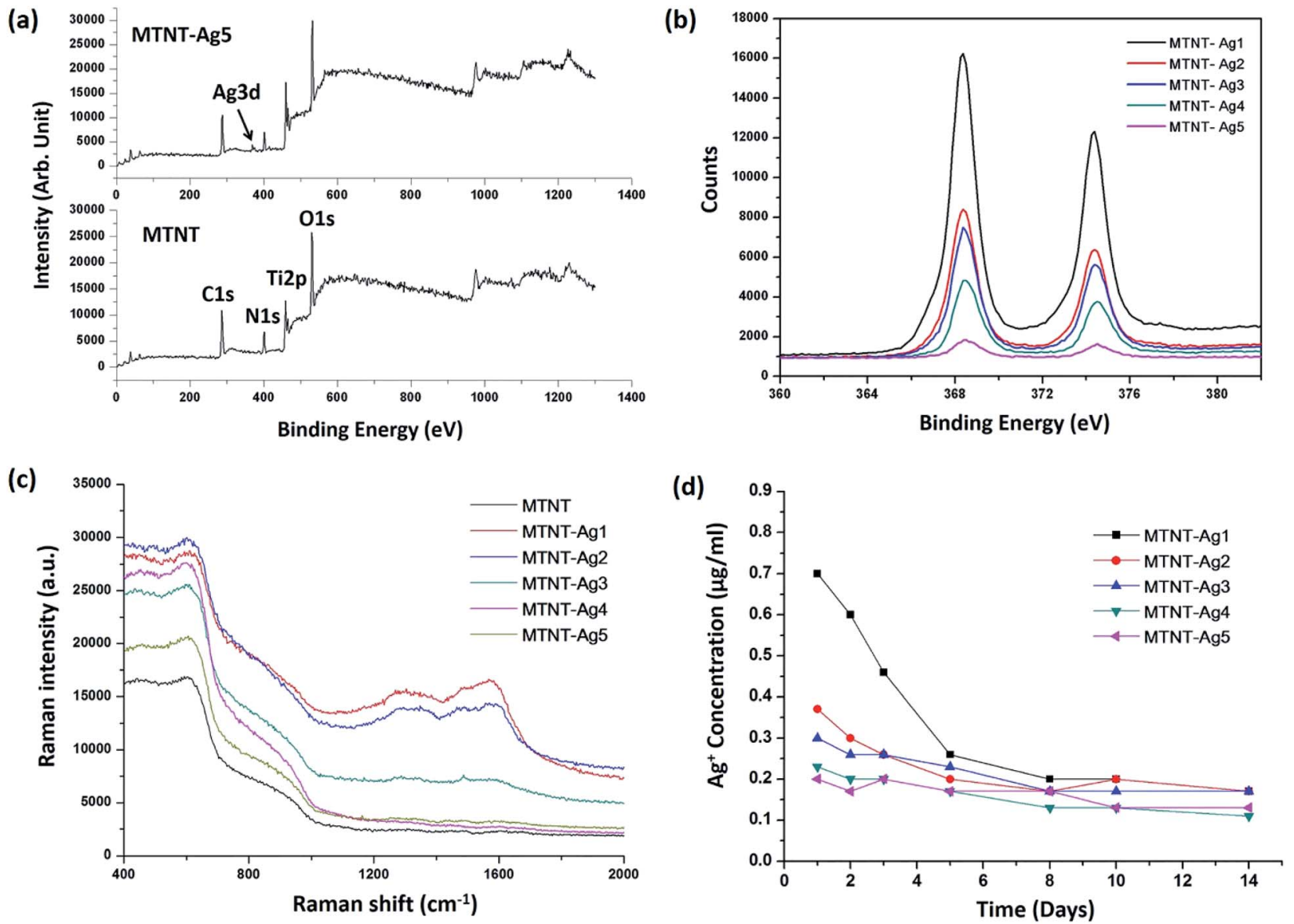

Fig. 4 (a) The survey XPS spectra of MTNT and MTNT-Ag5; (b) the high-resolution XPS spectra; and (c) Raman spectra for MTNT-Ag1 to MTNTAg5. (d) Non-cumulative silver release profiles from MTNT-Ag into PBS.

with all the antibacterial rates over $95 \%$, which were significantly high enough to eliminate potential infections. The MTNT-Ag5 sample had mild antibacterial effects at the rate of $11.33 \pm 1.82 \%$ on $S$. aureus and $42.86 \pm 4.57 \%$ on E. coli. The MTNT-Ag4 sample showed a moderate antibacterial effect on $S$. aureus (antibacterial rate was about $40 \%$ ), and a strong antibacterial effect on $E$. coli (antibacterial rate was over 95\%).

The topography of the attached bacteria was characterized via SEM after the samples were incubated with bacterial suspension and shaken for $6 \mathrm{~h}$. Clearly, more bacteria were adhered to the surface of MTNT than to those of other groups. Compared with the Ti group (set as a standard surface), there are relatively less bacteria adhered to the surface of the MTNTAg groups. The ruptured morphology imaged by SEM proved that some bacteria adhered to the MTNT-Ag surfaces had been damaged by AgNPs or silver ions released from the AgNPs (Fig. 5c, indicated with red arrows).

\subsection{Biocompatibility assay}

The cell viabilities on the samples were quantitatively measured by WST-1 after for 1, 3, 5, and 7 day culture, as shown in Fig. 6 b. There was no significant difference in the OD value among all samples incubated for one day. Compared with the Ti group, the MTNT group provided a more biocompatible surface during the entire culturing process, and the cell count on MTNT was significantly higher than that on Ti after 7 days of culture $(P<$ 0.05). Moreover, the MTNT-Ag samples exhibited different cell viability as compared to Ti. A significant difference in the WST-1 assay between Ti group and MTNT-Ag1 was observed after 3 days of culture $(P<0.05)$, and this difference lasted for 7 days; this indicates an obvious cytotoxicity for MTNT-Ag1. However, the samples MTNT-Ag2, MTNT-Ag3, MTNT-Ag4, and MTNT-Ag5 showed a good cell biocompatibility during the culturing process as there was no obvious difference in the WST-1 assay as compared to the Ti group $(P>0.05)$.

Although no statistically significant differences in the cell count among the sample groups were found after 1 day of culture, the cell configuration appeared different, as shown in Fig. 6a (day 1). The surfaces with MTNT-Ag1 and MTNT-Ag2 were unfavorable for preosteoblast spreading as the adherent cells were still spindle-shaped. It was noted that the cells on other sample groups adhered tightly to the substrates with much extended pseudopodia. With the extension of incubation time, cells, except for those in MTNT-Ag1 and MTNT-Ag2 groups, extensively spread and even secreted abundant extracellular matrix to benefit cell proliferation. After 7 days of culture, cell coverage on the surface decreased in the order of 
(a)
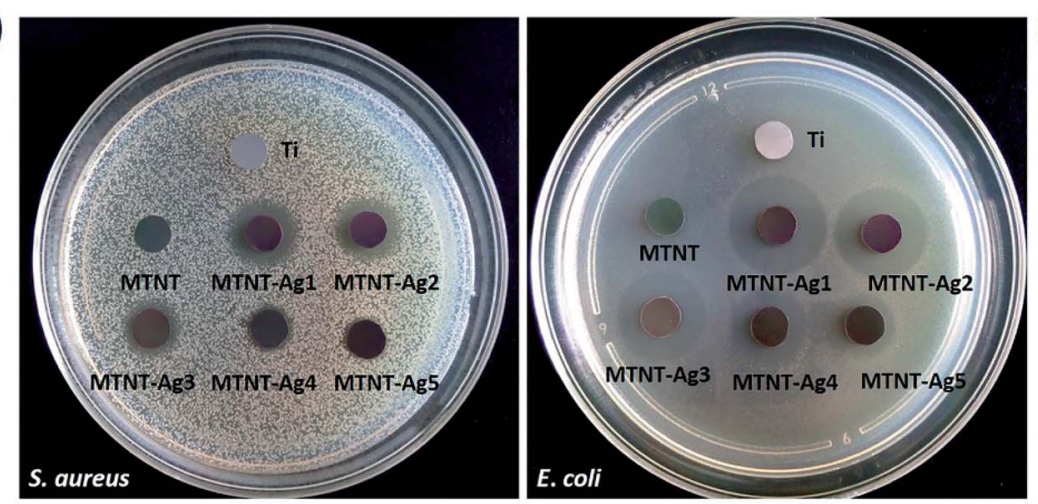

(b)

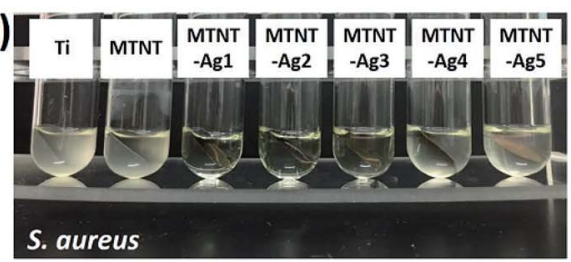

(c)
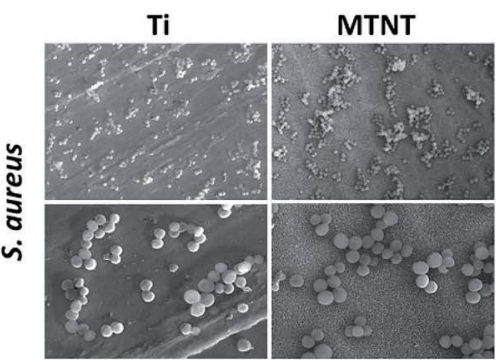

\section{MTNT-Ag1}
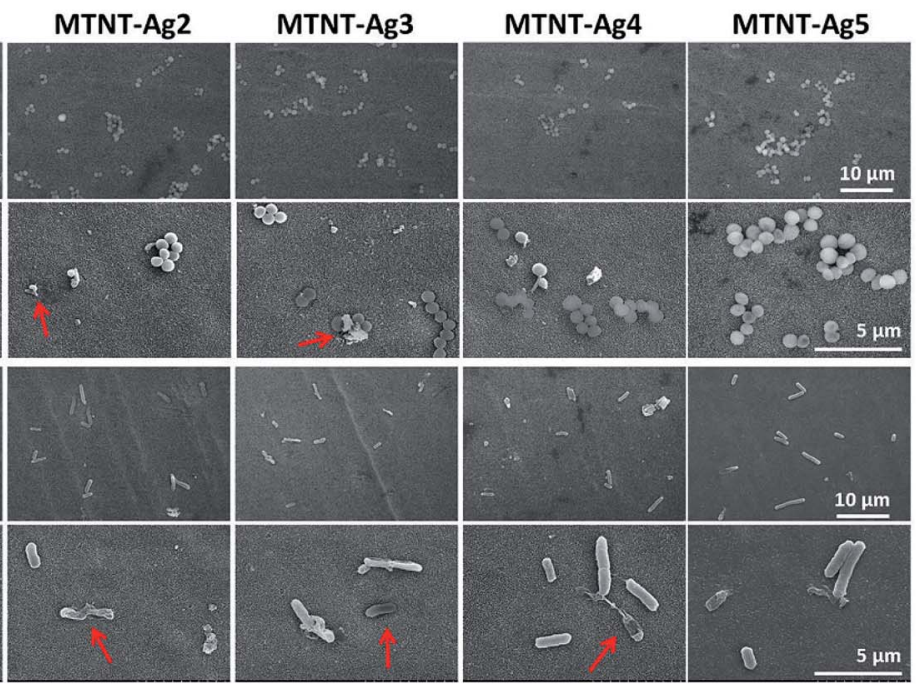

Fig. 5 The antibacterial activities of the MTNT-Ag samples: (a) the zone of inhibition assay; (b) the bactericidal efficiency test; and (c) the SEM images of bacteria on samples after incubation for $6 \mathrm{~h}$.

MTNT, Ti, MTNT-Ag5, MTNT-Ag4, MTNT-Ag3, MTNT-Ag2, and MTNT-Ag1.

The ALP activity was measured as an early marker for osteogenic differentiation potential of preosteoblasts (MC3T3-E1). As illustrated in Fig. 6c, only MTNT-Ag1 exhibited much lower ALP activity than Ti and other MTNT-Ag groups because of a higher number of $\mathrm{Ag}$ nanoparticles loaded on the surface. However, very similar ALP activity values were measured for the samples MTNT-Ag2, MTNT-Ag3, MTNT-Ag4, and MTNT-Ag5 after 10 days of culture; this indicated that there were no obvious negative effects on the osteogenic differentiation of the MC3T3-E1 cells.

After $24 \mathrm{~h}$ culture, the actin filament (F-actin) of preosteoblasts stained with FITC-phalloidin is shown in Fig. 7. Except the MTNT-Ag1 sample, the cells cultured on all samples had spread morphologies, the cytoskeleton was irregular with filaments extending in multiple directions, and the nuclei were defined round with homogeneous chromatin. However, MTNT$\mathrm{Ag} 1$ seemed to be unfavorable for the spreading of preosteoblasts; this was consistent with the results of the other biocompatibility evaluations.

\section{Discussion}

Upon implantation, large amount of proteins must immediately adsorb on the implant surface, and subsequently, the cells and bacterial adhesion and interaction to the surface occurs. There is a ubiquitous competition of adhesion between cells and bacteria on the implant surface, which is critical especially in the first several hours after implantation. It had been indicated that the quick adhesion of osteoblasts layer can actively protect implant surface from bacterial adhesion and biofilm formation. ${ }^{40-42}$ Implants with properties of fast osteoblast adhesion and effective antibacterial activity are therefore expected. Most of the previous studies have been focused on the preparation of antibacterial coatings with long-term antibacterial ability; ${ }^{16,22}$ herein, we have focused on constructing a bionic coating that is amiable for cell adhesion and effective in killing bacteria at the early stage.

As a universal adhesive protein derived from mussel Mytilus edulis, Mefp-1 has drawn significant attention in various fields such as in tissue adhesive and biomedical engineering. The adsorbed Mefp-1 coating usually has a thickness of several nanometers, ${ }^{28}$ and this thin protein film has a negligible 

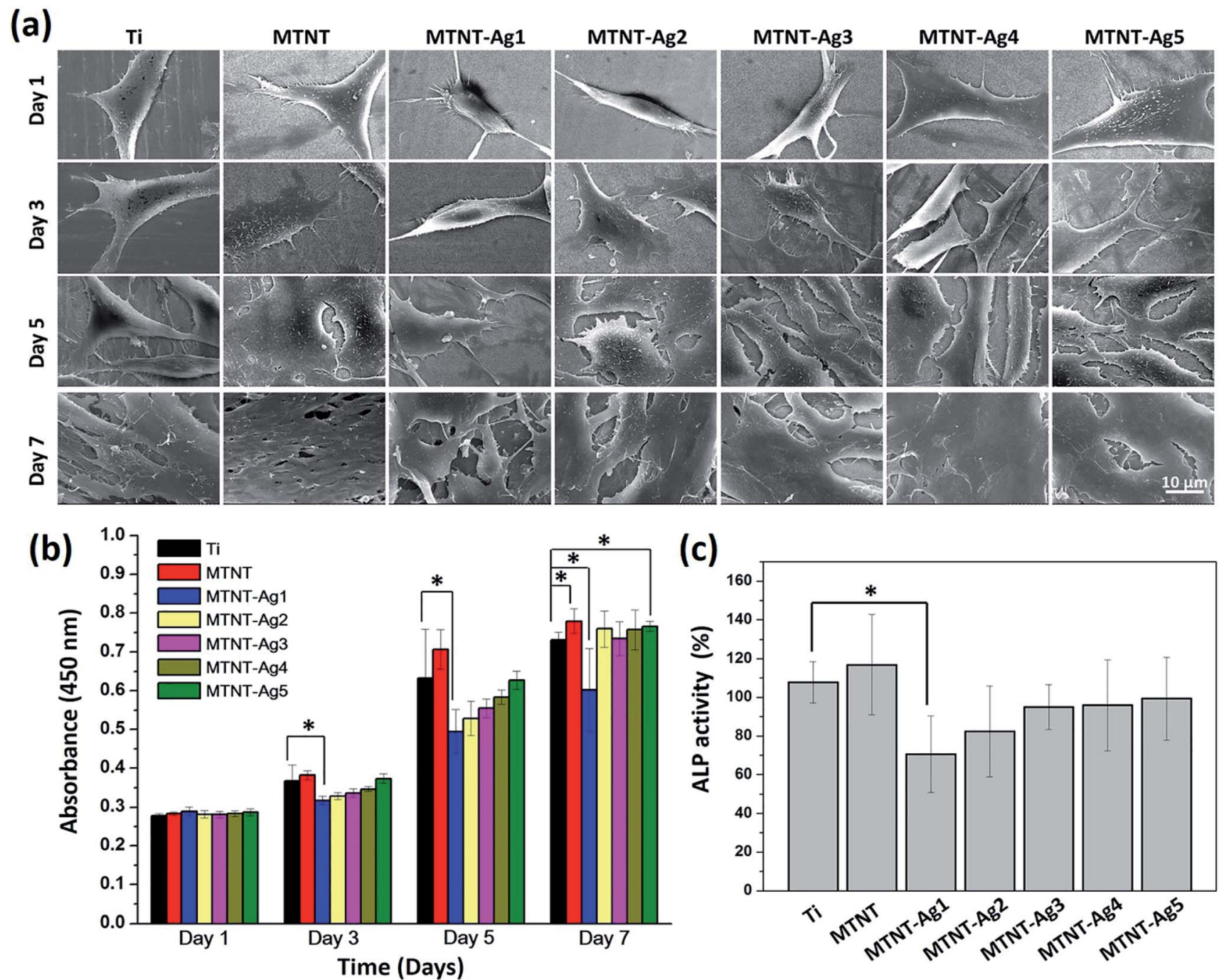

Fig. 6 (a) SEM images and (b) WST-1 assay for the proliferation of MC3T3-E1 cells on samples after incubation for 1, 3, 5, and 7 days; and (c) ALP activity assay after incubation for 10 days.

influence on the original surface morphology of TNTs, as shown in Fig. 2. The use of Mefp-1 had yielded a range of benefits: ${ }^{2,35,43-46}$ (i) nonspecific cell adhesion: the high content of
DOPA has endowed the Mefp-1 with excellent universal adhesive and cohesive properties. Moreover, Mefp-1 could form strong complexes with metal ions on the surfaces, which was
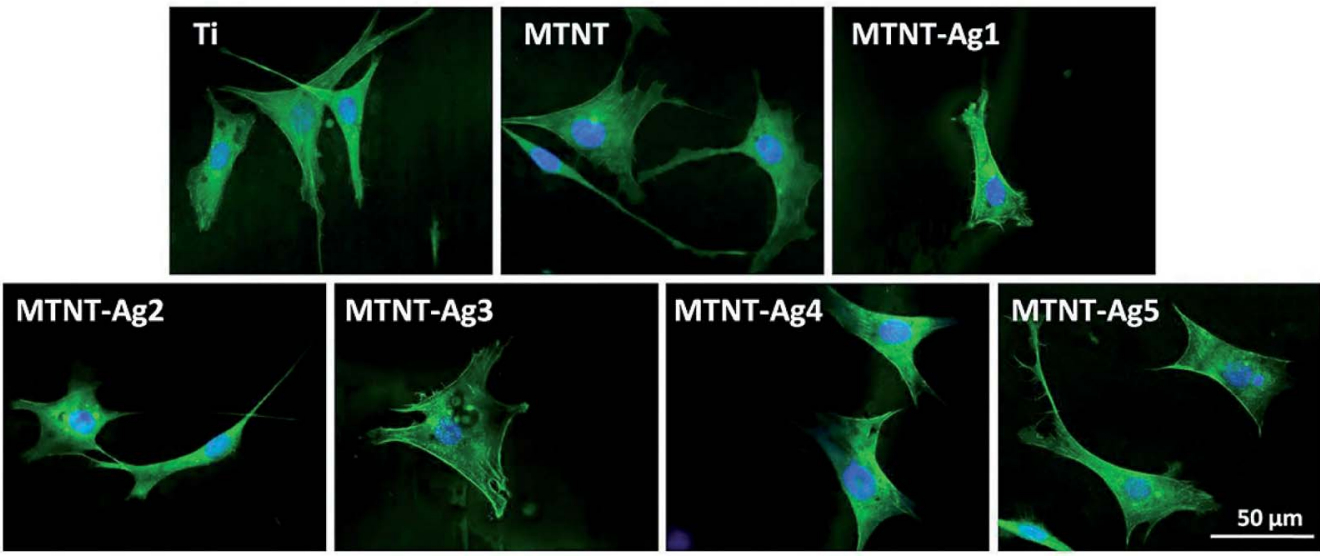

Fig. 7 Fluorescence images of MC3T3-E1 cell skeletons on samples after incubation for 1 day. 


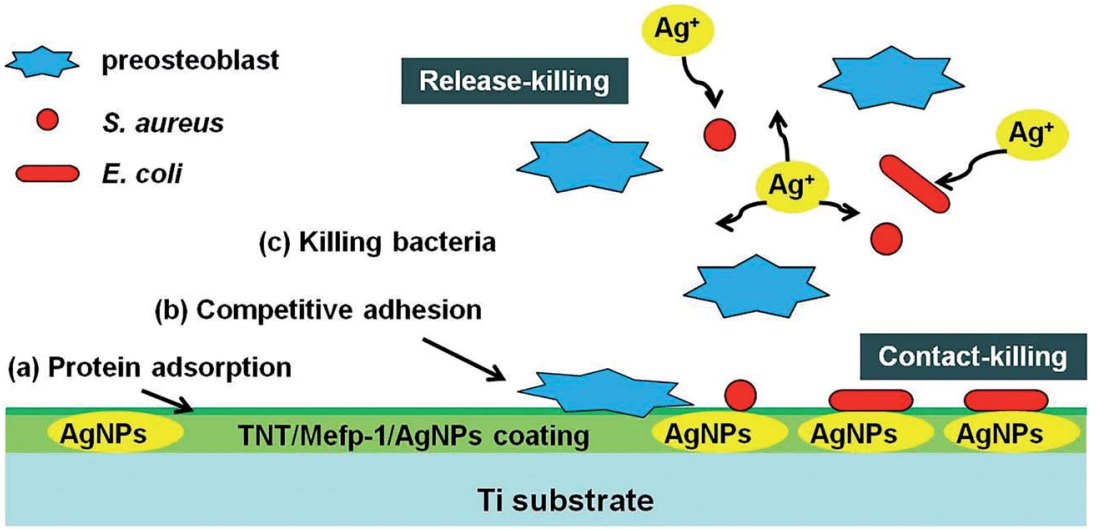

Fig. 8 Hypothetical representation of events at the TNT/Mefp-1/AgNP interface: (a) protein adsorption from blood and tissue fluids; (b) competitive adhesion between preosteoblasts and bacteria; and (c) a combination of release-killing and contact-killing processes.

able to enhance the protein adhesion even further. Glycoproteins and proteoglycans present on the cell membrane could form a weak binding with functional groups (such as positively charged Lys) on Mefp-1 through electrostatic interactions or van der Waals force; (ii) specific cell adhesion: the Mefp- 1 coatings exhibit latent reactivity towards catechol and amine or thiol groups via Schiff base base or Michael addition chemistry. Nucleophiles such as biomolecules and proteins could strongly attach to Mefp-1 coating through covalent interaction, which served as cell adhesion sites; (iii) surface hydrophilic property: hydrophilicity could be tuned by the deposited Mefp-1 coating, and hydrophilic substrates were generally favored for cell attachment. It is for the first time that Mefp- 1 coating has been used in surface antibacterial and biocompatible modifications based on its multifunctionality.

This very thin adherent Mefp-1 film could promote preosteoblast response and proliferation (Fig. 6a and b) and reduce AgNP-induced cytotoxicity to some extent. It is noteworthy that the size of the AgNPs constructed with assistance of Mefp- 1 is well confined at about $10 \mathrm{~nm}$ and independent of the silver nitrate concentration. Generally, the AgNPs produced through polydopamine films are uniformly distributed with a diameter in the range of $35-80 \mathrm{~nm}^{47-49}$ There exists a positive correlation between silver particle size and silver ions release, whereas AgNPs much easily release silver ions due to their active surface and high specific area. ${ }^{50}$ It has been reported that the AgNPs in smaller size range exhibit better antibacterial activity as compared to the large-sized AgNPs. ${ }^{51,52}$ Thus, the high antibacterial abilities of MTNT-Ag samples may be ascribed to the small size and uniform size distribution (as observed in SEM and TEM) of AgNPs anchored onto the nanotube wall. The TNT structure, on the other hand, provided a well-defined tubular nano-nest for supporting a sustainable silver ion release from the AgNPs. The dose of AgNPs embedded in the Mefp- 1 coating displayed an obvious correlation with the nitrate concentration and could be easily controlled in the experiments.

Although AgNPs have been widely used as inorganic antimicrobial agents, high cumulative concentration of leached silver ions from AgNPs coating was proven to produce apparent cytotoxicity ${ }^{16}$ Antibacterial property and cytocompatibility of
AgNPs prepared by different methods were compared, and the results are shown in Table S3. $\uparrow$ The loading dose, activity of AgNPs, and release rate of silver ions are essential factors determining the successful applications of AgNPs. ${ }^{22}$ AgNPs with properly controlled size and dose may be profitable. Finely designed AgNPs could support stem cells adhesion, enhance fibroblast attachment, and have no influence on osteoblast differentiation. ${ }^{53-55}$ The MTNT-Ag samples, such as MTNT-Ag2, MTNT-Ag3, MTNT-Ag4, and MTNT-Ag5 in this study, were cytocompatible during long period of cell culture when loaded with appropriate AgNP dose. The concomitant antibacterial ability could be effective in both Gram-positive and Gramnegative bacteria strains when the AgNP dose reached a certain level (MTNT-Ag3). Therefore, this suitable and precise loading architecture of AgNPs is able to balance the relationship between cytocompatibility and antibacterial ability. The controllable structure of TNT, Mefp-1 coating, AgNPs with confined size, and suitable dose are three pivotal elements of our TNT/Mefp-1/AgNP system. The hypothetical representation of events at the MTNT-Ag interface is described in Fig. 8. Large amounts of proteins from blood and tissue fluids were adsorbed on the modified implants. The preosteoblasts and planktonic bacteria were then competitively adhered to the surface. MTNT$\mathrm{Ag}$ coating served as a dual functional surface at the early stage, on which MC3T3-E1 cells were suitable to adhere and proliferate, and anchored bacteria were likely to be damaged. The AgNPs $(\sim 10 \mathrm{~nm})$ anchored on the surface of the nanotubes played a contact-killing role for both Gram-positive and Gramnegative bacteria (as shown in Fig. 5c with red arrows), whereas these particles may be too less to be effectively perceived by preosteoblasts (as shown in Fig. 6a). Moreover, the continuously releasing silver ions played a release-killing role for the planktonic bacteria.

Overall, this precise design and construction of MTNT-Ag coating on OIs assisted by Mefp- 1 are able to combine excellent antibacterial activity and cytocompatibility, indicating a promising potential in biomedical engineering. Further in vivo animal studies are needed for comprehensive evaluation before clinical applications. 


\section{Conclusions}

In this study, we successfully fabricated MTNT-Ag films via anodic oxidation and dip-coating methods assisted by the monolayered Mefp-1 coatings. Mefp-1 coating is powerful to improve the surface hydrophilic property, cell adhesive capacity, and in situ reduction of silver ions to uniform AgNPs onto TNT implants. It was found that the confined nanosize and suitable dose of AgNPs were crucial to endow the implants with both functions of antibacterial ability and cytocompatibility. In this regard, the preosteoblasts are more competitive than bacteria to adhere onto the MTNT-Ag surface as the surface environment can be deadly to bacteria and friendly to preosteoblasts. The high efficiency of the MTNT-Ag coating against Gram-positive and Gram-negative bacteria strains was attributed to AgNPs via both releasing-killing and contact-killing. The results of this study paved a way for multifunctional surface modification of medical devices with Mefp-1.

\section{Acknowledgements}

The authors acknowledge the financial support received from International S\&T Cooperation Program of China (No. 2014DFG52350), State Key Project of Research and Development (2016YFC1100301), National Natural Science Foundation of China (51571169), and Medical Scientific Youth Program Supported by Nanjing Command (18FBQN2014008).

\section{Notes and references}

1 A. Han, J. K. H. Tsoi, F. P. Rodrigues, J. G. Leprince and W. M. Palin, Int. J. Adhes. Adhes., 2016, 69, 58-71.

2 D. A. Puleo and A. Nanci, Biomaterials, 1999, 20, 2311-2321.

3 J. Liang, R. Song, Q. Huang, Y. Yang, L. Lin, Y. Zhang, P. Jiang, H. Duan, X. Dong and C. Lin, Electrochim. Acta, 2015, 174, 1149-1159.

4 Y. Wang, H. Yu, C. Chen and Z. Zhao, Mater. Des., 2015, 85, 640-652.

5 S. D. Puckett, E. Taylor, T. Raimondo and T. J. Webster, Biomaterials, 2010, 31, 706-713.

6 P. L. Jiang, J. H. Liang, R. Song, Y. M. Zhang, L. Ren, L. H. Zhang, P. F. Tang and C. J. Lin, ACS Appl. Mater. Interfaces, 2015, 7, 14384-14396.

7 Y.-S. Sun, J.-H. Chang and H.-H. Huang, Surf. Coat. Technol., 2016, 303, 289-297.

8 T. Sakurai, M. Yoshinari, T. Toyama, T. Hayakawa and C. Ohkubo, J. Biomed. Mater. Res., Part A, 2016, 104, 15001509.

9 K. Vasilev, J. Cook and H. J. Griesser, Expert Rev. Med. Devices, 2009, 6, 553-567.

10 R. Kargupta, S. Bok, C. M. Darr, B. D. Crist, K. Gangopadhyay, S. Gangopadhyay and S. Sengupta, Wiley Interdiscip. Rev.: Nanomed. Nanobiotechnol., 2014, 6, 475495.

11 S. Ferraris and S. Spriano, Mater. Sci. Eng., C, 2016, 61, 965978.
12 C.-K. Wei and S.-J. Ding, J. Mater. Chem. B, 2017, 5, 19431953.

13 C. Marambio-Jones and E. M. V. Hoek, J. Nanopart. Res., 2010, 12, 1531-1551.

14 D. J. Stickler, Nat. Clin. Pract. Urol., 2008, 5, 598-608.

15 O. V. Babkina, V. A. Svetlichnyi, I. N. Lapin, V. T. Novikov and

A. L. Nemoikina, Russ. Phys. J., 2013, 56, 405-410.

16 L. Zhao, H. Wang, K. Huo, L. Cui, W. Zhang, H. Ni, Y. Zhang, Z. Wu and P. K. Chu, Biomaterials, 2011, 32, 5706-5716.

17 Y. Lai, H. Zhuang, K. Xie, D. Gong, Y. Tang, L. Sun, C. Lin and Z. Chen, New J. Chem., 2010, 34, 1335-1340.

18 H. Qin, H. Cao, Y. Zhao, C. Zhu, T. Cheng, Q. Wang, X. Peng, M. Cheng, J. Wang, G. Jin, Y. Jiang, X. Zhang, X. Liu and P. K. Chu, Biomaterials, 2014, 35, 9114-9125.

19 D. E. Fullenkamp, J. G. Rivera, Y.-k. Gong, K. A. Lau, L. He, R. Varshney and P. B. Messersmith, Biomaterials, 2012, 33, 3783-3791.

20 H. Xu, X. Shi, H. Ma, Y. Lv, L. Zhang and Z. Mao, Appl. Surf. Sci., 2011, 257, 6799-6803.

21 C. Falentin-Daudré, E. Faure, T. Svaldo-Lanero, F. Farina, C. Jérôme, C. Van De Weerdt, J. Martial, A.-S. Duwez and C. Detrembleur, Langmuir, 2012, 28, 7233-7241.

22 Z. J. Jia, P. Xiu, M. Li, X. C. Xu, Y. Y. Shi, Y. Cheng, S. C. Wei, Y. F. Zheng, T. F. Xi, H. Cai and Z. J. Liu, Biomaterials, 2016, 75, 203-222.

23 J. Ren, P. Han, H. Wei and L. Jia, ACS Appl. Mater. Interfaces, 2014, 6, 3829-3838.

$24 \mathrm{~K} . \mathrm{Wu}, \mathrm{Y}$. Yang, Y. Zhang, J. Deng and C. Lin, Int. J. Nanomed., 2015, 10, 7241-7252.

25 Y. Cong, T. Xia, M. Zou, Z. Li, B. Peng, D. Guo and Z. Deng, J. Mater. Chem. B, 2014, 2, 3450-3461.

26 Q. Lu, E. Danner, J. H. Waite, J. N. Israelachvili, H. Zeng and D. S. Hwang, Biomaterials, 2012, 33, 1903-1911.

27 Q. Lin, D. Gourdon, C. Sun, N. Holten-Andersen, T. H. Anderson, J. H. Waite and J. N. Israelachvili, Proc. Natl. Acad. Sci. U. S. A., 2007, 104, 3782-3786.

28 F. Zhang, M. Sababi, T. Brinck, D. Persson, J. Pan and P. M. Claesson, J. Colloid Interface Sci., 2013, 404, 62-71.

29 D. S. Hwang, S. B. Sim and H. J. Cha, Biomaterials, 2007, 28, 4039-4046.

30 R. Appeltant, D. Maes and A. Van Soom, Anal. Biochem., 2015, 480, 31-33.

31 X. Chen and W. Zhang, Chem. Soc. Rev., 2017, 46, 734-760. 32 V. B. Damodaran, D. Bhatnagar, V. Leszczak and K. C. Popat, RSC Adv., 2015, 5, 37149-37171.

33 E. Yamamoto and K. Kuroda, Bull. Chem. Soc. Jpn., 2016, 89, 501-539.

34 K. Ariga, J. Li, J. Fei, Q. Ji and J. P. Hill, Adv. Mater., 2016, 28, 1251-1286.

35 M. R. Souza, K. M. Reyes, N. T. C. Oliveira, N. K. Kuromoto and C. E. B. Marino, Mater. Res. Express, 2016, 3, 1-7.

36 K. F. Huo, B. Gao, J. J. Fu, L. Z. Zhao and P. K. Chu, RSC Adv., 2014, 4, 17300-17324.

37 Q. Wang, J.-Y. Huang, H.-Q. Li, Z. Chen, A. Z.-J. Zhao, Y. Wang, K.-Q. Zhang, H.-T. Sun, S. S. Al-Deyab and Y.-K. Lai, Int. J. Nanomed., 2016, 11, 4819-4834. 
38 F. Zhang, T. Brinck, B. D. Brandner, P. M. Claesson, A. Dedinaite and J. Pan, Electrochim. Acta, 2013, 107, 276291.

39 M. Sababi, F. Zhang, O. Krivosheeva, M. Forslund, J. Pan, P. M. Claesson and A. Dedinaite, J. Electrochem. Soc., 2012, 159, C364-C371.

40 S. Veerachamy, T. Yarlagadda, G. Manivasagam and P. Yarlagadda, Proc. Inst. Mech. Eng., Part H, 2014, 228, 1083-1099.

41 P. Klemm, R. M. Vejborg and V. Hancock, Appl. Microbiol. Biotechnol., 2010, 88, 451-459.

42 Y. H. An and R. J. Friedman, J. Biomed. Mater. Res., 1998, 43, 338-348.

43 H. Lee, B. P. Lee and P. B. Messersmith, Nature, 2007, 448, 338-U334.

44 H. Lee, S. M. Dellatore, W. M. Miller and P. B. Messersmith, science, 2007, 318, 426-430.

45 H. Lee, J. Rho and P. B. Messersmith, Adv. Mater., 2009, 21, 431-434.

46 S. H. Ku and C. B. Park, Biomaterials, 2010, 31, 9431-9437.

47 M. Sureshkumar, P.-N. Lee and C.-K. Lee, J. Mater. Chem., 2011, 21, 12316-12320.
48 M. Sureshkumar, D. Y. Siswanto, Y.-C. Chen, C.-K. Lee and M.-J. Wang, J. Polym. Sci., Part B: Polym. Phys., 2013, 51, 303-310.

49 Z. J. Jia, P. Xiu, P. Xiong, W. H. Zhou, Y. Cheng, S. C. Wei, Y. F. Zheng, T. F. Xi, H. Cai, Z. J. Liu, C. M. Wang, W. P. Zhang and Z. J. Li, ACS Appl. Mater. Interfaces, 2016, 8, 28495-28510.

50 C. E. Albers, W. Hofstetter, K. A. Siebenrock, R. Landmann and F. M. Klenke, Nanotoxicology, 2013, 7, 30-36.

51 M. Paul, K. P. Prasad, G. Rathore, K. Kumar and R. Sharma, Indian J. Anim. Sci., 2016, 86, 964-971.

52 X. Chen, X. Huang, C. Zheng, Y. Liu, T. Xu and J. Liu, J. Mater. Chem. B, 2015, 3, 7020-7029.

53 C. Zhu, N. R. Bao, S. Chen and J. N. Zhao, Appl. Surf. Sci., 2016, 389, 7-16.

54 S.-h. Hsu, H.-J. Tseng and Y.-C. Lin, Biomaterials, 2010, 31, 6796-6808.

55 L. Pauksch, S. Hartmann, M. Rohnke, G. Szalay, V. Alt, R. Schnettler and K. S. Lips, Acta Biomater., 2014, 10, 439449. 